\title{
Model-Based Performance Analysis of Service-Oriented Systems (Invited Talk)
}

\author{
Dorina C. Petriu \\ Department of Systems and Computer Engineering, \\ Carleton University, Ottawa, Ontario, Canada \\ http://www.sce.carleton.ca/faculty/petriu.html
}

\begin{abstract}
Quantitative performance analysis of service-oriented systems can be conducted in the early development phases by transforming a UML software model extended with performance annotations into a performance model (such as queueing networks, Petri nets, stochastic process algebra) which can be solved with existing performance analysis tools. The OMG standard "UML Profile for Modeling and Analysis of Real-Time and Embedded systems (MARTE)" can be used for adding performance annotations to a given UML model.

The talk discussed the type of MARTE performance annotations and the principles for transforming annotated software models into performance models. Such a transformation must bridge a large semantic gap between the source and target model for two main reasons: performance models concentrate on resource usage and abstract away many details of the original software model, and the performance model requires platform information which is not contained in the software application model. The starting point for the performance model derivation is a platform independent model (PIM) of a SOA system representing the process workflows, architecture of the components offering services, and behavior of the runtime scenarios chosen for evaluation. The PIM is transformed into a platform specific model (PSM) by weaving platform services through aspect-oriented modeling techniques. Other research challenges will be also discussed, such as merging performance modeling and measurements and analyzing performance effects of SOA design patterns.
\end{abstract}

